\title{
The Impact of Ulcerative Colitis on Colorectal Cancer Prognosis: The Jury is Still Out
}

\author{
Arielle Kanters, MD, MS, and David Liska, MD \\ Department of Colorectal Surgery, Digestive Disease \& Surgery Institute, Cleveland Clinic, Cleveland
}

The increased risk of developing colorectal cancer in the inflammatory bowel disease (IBD) population is well established, and previous work has demonstrated a 30-year cumulative risk of $18 \%$ in ulcerative colitis patients. ${ }^{1}$ This risk is specifically associated with presence of primary sclerosing cholangitis, extent and duration of disease, and family history. ${ }^{1}$ With the more recent advancements in management options (including enhanced therapies and more rigorous surveillance regimens), the incidence of ulcerative colitis-associated colorectal cancer (UC-CRC) is decreasing, but it remains an area of focus to identify opportunities to further improve outcomes. While risk factors, carcinogenesis, and clinical presentation of UC$\mathrm{CRC}$ are known to be different from sporadic colorectal cancer (s-CRC), it remains unclear if these differences also portend a different prognosis in terms of long-term oncologic outcomes. ${ }^{1,2}$ This uncertainty is related to previous studies being limited by a small number of patients or incompletely characterized oncologic outcomes.

Lin et al. have taken on the task of comprehensively evaluating long-term oncologic outcomes in UC-CRC versus s-CRC by studying patients in the Danish National Patient Register undergoing curative-intent surgery for CRC over a period of 12 years, including 222 patients with UC-CRC that were propensity score matched to 1110 patients with s-CRC. ${ }^{3}$ After matching for disease stage and other confounding factors (age, gender, comorbidities, and surgical technique), the authors found no significant

(C) Society of Surgical Oncology 2022

First Received: 7 December 2021

Accepted: 25 December 2021;

Published Online: 11 January 2022

D. Liska, MD

e-mail: liskad@ccf.org difference in disease-free survival, recurrence-free survival, or all-cause mortality between UC-CRC and s-CRC. They further validated their findings in the context of existing literature by performing a systematic review with meta-analysis and again found no significant difference in outcomes between the two groups, although there was substantial heterogeneity between included studies with regard to all-cause mortality.

We must applaud the authors on their clearly defined outcomes and rigorous approach to a matched analysis as it helps determine if there are differences in CRC outcomes related to UC once adjusting for confounding factors. Without proper adjustments for these variables, some previous publications demonstrating worse survival in UC patients could be attributed to delayed diagnosis or mortality related to comorbid conditions rather than more aggressive tumor biology in UC. The equivalent mortality rates by stage at diagnosis in both groups suggest that, despite differences in carcinogenesis, cancer outcomes following surgery are similar for UC-CRC and s-CRC.

However, in contrast to these data from Denmark, a recently published study on this topic (not included in the author's meta-analysis) by Arhi et al. analyzed the outcomes of UC-CRC using the English National Cancer Registry. ${ }^{4}$ In this larger cohort, UC-CRC $(n=1922)$ outcomes were compared with s-CRC $(n=231,102)$ when stratifying patients according to stage. After adjusting for confounding factors (including age, gender, and comorbidities) this study found that, in patients with stage III or IV disease, UC-CRC patients had significantly worse survival compared with s-CRC patients. These two recent national database studies exemplify the conflicting results that have been published on this topic to date. Due to heterogeneous study design with different inclusion criteria and outcomes analyzed, several publications are not accounted for in the meta-analysis included by Lin et al. in the current study. ${ }^{4-6}$ 
The discrepancies in outcomes emphasize that we still do not fully understand the natural history of cancer in the IBD population and raise the point that, to truly investigate this pathology, we must develop a standardized approach to study design. Further work must clearly define inclusion criteria and outcomes to allow for pooled analyses. For example, we see in this review that less than one-third of the UC-CRC patients underwent a total colectomy or proctocolectomy. It is generally recommended that a patient with malignant lesions in the setting of IBD undergoes a total colectomy given the risk of metachronous cancer, ${ }^{7,8}$ yet there are no strict guidelines that dictate this management strategy. ${ }^{9,10}$ Olén et al. found that patients with extensive colitis had a higher risk of developing colorectal cancer than those with isolated left-sided colitis or proctitis. ${ }^{1}$ This raises the question of whether patients with segmental disease (e.g., left-sided colitis) should be analyzed separately from those with pancolitis. Perhaps only those with mild disease have similar outcomes to the s-CRC population. It is challenging to compare these populations when we do not account for the underlying extent of disease. Prospective studies to better guide optimal resection parameters are needed to develop informed guidelines.

There is increasing evidence demonstrating that IBDCRC develops via a different oncologic pathway than s-CRC. Thus far we have seen that IBD-CRC has lower rates of APC and KRAS mutations and earlier p53 mutations compared with sCRC. ${ }^{11}$ There are also higher rates of genome-wide methylation and decreased genetic changes related to age-driven transition mutations. This may be responsible for the earlier age of onset and differences in tumor distribution linked to inflammation. ${ }^{12}$ Epigenetic differences between IBD-CRC and s-CRC may also play a role in long-term prognosis as IBD-CRC have demonstrated higher rates of WNT pathway dysregulation causing mesenchymal type tumors. These tumors are in turn associated with drug resistance and decreased overall survival. ${ }^{12}$ Ideally, future studies examining differences between IBD-CRC and s-CRC would not only include relevant clinical data regarding severity and extent of disease but also a comparison of molecular phenotypes to allow for a more comprehensive understanding and facilitate the development of novel targeted therapies.

Despite this lack of clarity, it does appear that recent advances in the approach to surveillance and treatment of UC have led to an improvement in overall survival. It is imperative that we continue to emphasize in our patients the importance of rigorous surveillance according to established guidelines and management of dysplasia to allow for prevention and early diagnosis UC-CRC. ${ }^{13}$ To take the next steps towards improving patient outcomes, we must now focus our efforts on teasing out the underlying biologic reasons for this increased risk of cancer. Answering these additional questions will allow for tailored management options on the individual patient level with the ultimate goal of improved patient survival.

\section{REFERENCES}

1. Olén O, Erichsen R, Sachs MC, Pedersen L, Halfvarson J, Askling $\mathrm{J}$, et al. Colorectal cancer in ulcerative colitis: a Scandinavian population-based cohort study. Lancet (London, England). 2020;395(10218):123-31.

2. Jensen AB, Larsen M, Gislum M, Skriver MV, Jepsen P, Nørgaard B, et al. Survival after colorectal cancer in patients with ulcerative colitis: a nationwide population-based Danish study. Am J Gastroenterol. 2006;101(6):1283-7.

3. Lin V, Lohse R, Madsen M, Fransgaard T, Remzi F, Gögenur I. Long-term outcomes after colorectal surgery in patients with ulcerative colitis-associated colorectal cancer versus sporadic colorectal cancer. Ann Surg Oncol. 2021. https://doi.org/10.1245/ s10434-021-10759-8.

4. Arhi C, Askari A, Nachiappan S, Bottle A, Arebi N, Athanasiou $\mathrm{T}$, et al. Stage at diagnosis and survival of colorectal cancer with or without underlying inflammatory bowel disease: a populationbased study. J Crohn's Colitis. 2021;15(3):375-82.

5. Watanabe T, Konishi T, Kishimoto J, Kotake K, Muto T, Sugihara K. Ulcerative colitis-associated colorectal cancer shows a poorer survival than sporadic colorectal cancer: a nationwide Japanese study. Inflamm Bowel Dis. 2011;17(3):802-8.

6. Gearhart SL, Nathan H, Pawlik TM, Wick E, Efron J, Shore AD. Outcomes from IBD-associated and non-IBD-associated colorectal cancer: a Surveillance Epidemiology and End Results Medicare study. Dis Colon Rectum. 2012;55(3):270-7.

7. Clarke WT, Feuerstein JD. Colorectal cancer surveillance in inflammatory bowel disease: Practice guidelines and recent developments. World J Gastroenterol. 2019;25(30):4148-57.

8. Annese V, Beaugerie L, Egan L, Biancone L, Bolling C, Brandts $\mathrm{C}$, et al. European evidence-based consensus: inflammatory bowel disease and malignancies. $J$ Crohn's Colitis. 2015;9(11):945-65.

9. Rubin DT, Ananthakrishnan AN, Siegel CA, Sauer BG, Long MD. ACG Clinical Guideline: ulcerative colitis in adults. Am J Gastroenterol. 2019;114(3):384-413.

10. Krugliak Cleveland N, Ollech JE, Colman RJ, Rodriquez D, Hirsch A, Cohen RD, et al. Efficacy and follow-up of segmental or subtotal colectomy in patients with colitis-associated neoplasia. Clin Gastroenterol Hepatol. 2019;17(1):205-6.

11. Pekow J, Hernandez K, Meckel K, Deng Z, Haider HI, Khalil A, et al. IBD-associated Colon cancers differ in DNA methylation and gene expression profiles compared with sporadic colon cancers. J Crohn's Colitis. 2019;13(7):884-93.

12. Rajamäki K, Taira A, Katainen R, Välimäki N, Kuosmanen A, Plaketti RM, et al. Genetic and epigenetic characteristics of inflammatory bowel disease-associated colorectal cancer. Gastroenterology. 2021;161(2):592-607.

13. Laine L, Kaltenbach T, Barkun A, McQuaid KR, Subramanian V, Soetikno R. SCENIC international consensus statement on surveillance and management of dysplasia in inflammatory bowel disease. Gastroenterology. 2015;148(3):639-51.e28.

Publisher's Note Springer Nature remains neutral with regard to jurisdictional claims in published maps and institutional affiliations. 